$\mathrm{E}$

EVALUAR
2019, Vol. 19, No. 2

ISSN 1667-4545

Recuperado de https://revistas.unc.edu.ar/index.php/revaluar

Laboratorio de Evaluación Psicológica y Educativa

Facultad de Psicología - Universidad Nacional de Córdoba

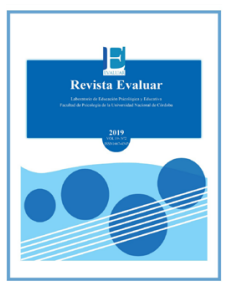

\title{
Validación de una versión breve de la Escala de Satisfacción Sexual Subjetiva (ESSS-B) en Puerto Rico
}

\section{Validation of a Short Version of the Subjective Sexual Satisfaction Scale (ESSS-B) in Puerto Rico}

\author{
Juan Aníbal González-Rivera * 1, Idania Hernández-Gato ${ }^{2}$ \\ 1 - School of Behavioral and Brain Sciences, Ponce Health Sciences University, San Juan University Center, Puerto Rico. \\ 2 - Universidad Carlos Albizu, San Juan Campus, Puerto Rico.
}

Recibido: 17/01/2019 Revisado: 25/02/2019 Aceptado: 10/03/2019
Introducción

Estudio 1

Método

Resultados

Estudio 2

Método

Resultados

Discusión

Referencias

\section{Resumen}

El objetivo principal de este proyecto fue desarrollar una versión breve de la Escala de Satisfacción Sexual Subjetiva. Para ello, se realizaron dos estudios independientes. El primero empleó una muestra de 200 participantes para analizar la confiabilidad y la estructura factorial de la escala con criterios estrictos de selección de ítems. En el segundo, se utilizó una muestra de 275 participantes, se efectuó un análisis factorial confirmatorio y se calculó la validez convergente y concurrente de la versión breve del instrumento (ESSS-B). Los resultados de los estudios mostraron propiedades psicométricas adecuadas en la escala. El índice de confiabilidad alfa de Cronbach de la escala fue .91. Los siete ítems de la ESSS-B cumplieron con los criterios de discriminación. Se realizaron análisis de validez convergente y concurrente, de los que se obtuvieron valores estadísticamente significativos. Estos resultados sugieren que la ESSS-B tiene el potencial para medir adecuadamente la satisfacción sexual de una forma global y subjetiva en adultos puertorriqueños.

Palabras clave: satisfacción sexual, sexualidad, propiedades psicométricas, validación

\section{Summary}

The objective of this study was to develop a short version of the Subjective Sexual Satisfaction Scale. Two independent studies were carried out with this purpose. The first study, of 200 participants, analyzed the reliability and factorial structure of the scale with strict criteria of item selection. In the second study, of 275 participants, a confirmatory factorial analysis was carried out and the convergent and concurrent validity of the short version of the instrument (ESSS-B) was calculated. The results of the studies showed adequate psychometric properties in the scale. The reliability index of the scale was of .91 (Cronbach's alpha). The seven items of the ESSS-B complied with the discrimination criteria. Convergent and concurrent validity analyses were performed, obtaining statistically significant values. These results suggest that the ESSS-B has the potential to adequately measure sexual satisfaction in a global and subjective way among Puerto Rican adults.

Keywords: sexual satisfaction, sexuality, psychometric properties, validation

*Correspondencia a: Dr. Juan A. González-Rivera, 500 West Main Suite 215, Bayamón, Puerto Rico, 00961. Tel.: 011 787 315 6034. jagonzalez@psm.edu Nota de Autor: El autor expresa que no hubo conflictos de intereses al redactar el manuscrito. 


\section{Introducción}

La salud sexual tiene un rol fundamental en la salud general, en el bienestar y en la calidad de vida de un individuo. La misma ha sido definida como "la integración de los aspectos somáticos, emocionales, intelectuales y sociales de un ser humano sexual en formas que enriquecen y mejoran la personalidad, la comunicación y el amor" (World Health Organization, 2010). A su vez, la satisfacción sexual ha sido identificada como uno de los factores principales de la salud sexual. Asimismo, las dificultades sexuales pueden ocasionar falta de comunicación y conflicto en las parejas, lo cual está altamente relacionado con la disminución en la satisfacción con la relación (Metz \& Epstein, 2002; Rowland, van Diest, Incrocci, \& Slob, 2005).

Aunque en otras culturas existen instrumentos que permiten evaluar el constructo satisfacción sexual, González-Rivera, Veray-Alicea, Santiago-Santos, Castro-Castro, y Quiñones-Soto (2017) contribuyeron a su investigación científica en Puerto Rico al crear el primer instrumento que permite medir la satisfacción sexual tomando en consideración a la población masculina. En ese momento solo existía el Inventario de Satisfacción Sexual para Mujeres creado por Vizcarrondo-Godreau (2001), el cual limita la evaluación de este constructo a la población femenina puertorriqueña. Los investigadores desarrollaron y validaron la Escala de Satisfacción Sexual Subjetiva (ESSS) con el propósito de ofrecer un indicador general de satisfacción sexual para ser utilizado con fines investigativos y académicos, así como medida de avalúo general en los procesos de psicoterapia.

No obstante, ante la necesidad de tener disponibles instrumentos breves, de fácil administración y con adecuados índices de validez y consistencia interna -como recomienda DeRogatis
(2008) - que favorezcan la medición de la satisfacción sexual en investigaciones académicas, este estudio tiene el propósito de desarrollar una versión breve de la ESSS, ya que un instrumento con estas cualidades facilitaría la tarea investigativa de temas asociados al conflicto y la satisfacción en las relaciones de pareja.

\section{Satisfacción sexual subjetiva}

La mayoría de los estudios de satisfacción sexual fueron realizados durante la década de los 90 (Darling, Davidson, \& Jennings, 1991; Lawrance \& Byers, 1995; Orbuch \& Harvey, 1991; Peplau, Cochran, \& Mays, 1997; Renaud, Byers, \& Pan, 1997; Young, Denny, Luquis, \& Young, 1998). A pesar de que el interés en este campo fue aumentando durante las pasadas dos décadas y estudios más recientes han destacado la importancia de la satisfacción sexual en la calidad de vida de los individuos (Ahumada, Lüttges, Molina, \& Torres, 2014; Davison, Bell, LaChina, Holden, \& Davis, 2009), aún queda mucho por explorar acerca de las implicaciones de este constructo desde una perspectiva holística.

Algunas definiciones de satisfacción sexual tienen una denotación poco precisa. Esto se debe a que los investigadores han utilizado diferentes enfoques para evaluar este constructo y las estrategias de medición varían de acuerdo con los posibles indicadores que podrían afectar la vida sexual de un individuo (Monteiro-Pascoal, Narciso, \& Monteiro-Pereira, 2014). Varios modelos teóricos han revelado hallazgos que han sido inconsistentes con los significados operacionales (DeLamater \& Hyde, 2004; Sprecher \& Cate, 2004). De acuerdo con la estructura de cuatro factores examinada por Hassebrauck y Fehr (2002), la sexualidad representa una de las cuatro dimensiones de la satisfacción de la relación. Los tres facto- 
res restantes examinados en este estudio fueron la intimidad, la capacidad para llegar a acuerdos y la independencia. Estos postulados son consistentes con el análisis realizado por Hurlbert, Apt y Rabehl (1993), quienes enfatizaron la importancia de la intimidad y la comunicación en la relación al evaluar la satisfacción sexual en general.

Así como ocurre con muchas definiciones de satisfacción sexual y bienestar, el constructo satisfacción sexual también es medido de una manera subjetiva, ya que está respaldado por un conjunto de experiencias personales (McClelland, 2010). Dicho esto, la satisfacción sexual ha sido definida como "una respuesta afectiva que surge de la propia evaluación subjetiva de las dimensiones positivas y negativas asociadas a la propia relación sexual" (Lawrance \& Byers, 1995, p. 268). Las personas tienden a calificar sus niveles de satisfacción sexual al analizar el modo en que la actividad sexual cumple con sus expectativas individuales (DeLamater, 1991). Las circunstancias sociales como el sexismo, el estigma sexual y el ambiente cultural son tomadas en consideración al comparar las recompensas sexuales y la percepción de cómo debe ser una relación sexual (Lawrance \& Byers, 1995; McClelland, 2010).

La satisfacción sexual ha sido fuertemente vinculada a la satisfacción de la relación, dado que está relacionada con la felicidad que una persona expresa sentir en el aspecto sexual de su relación (Pascoal et al., 2014; Sprecher \& Cate, 2004). Algunos estudios han demostrado que las variables satisfacción de la relación, amor y compromiso correlacionan positivamente con la satisfacción sexual tanto en hombres como en mujeres (Sprecher, 2002; Stephenson, Ahrold, \& Meston, 2011). Estudios realizados con parejas en relaciones a largo plazo (el 50\% de ellas tienen más de 25 años de duración) revelaron discrepancias entre lo manifestado por cada uno de los miembros de la pareja. Las mujeres expresaron sentir- se más satisfechas sexualmente, mientras que los hombres manifestaron mayor felicidad con la relación (Heiman et al., 2011). A pesar de que los hallazgos relacionados al género no son consistentes, ya que algunos estudios han demostrado diferencias entre hombres y mujeres mientras que otros no, la tendencia a percibir una mayor satisfacción sexual está vinculada con los otros aspectos emocionales que se obtienen al estar comprometidos en una relación (Sánchez-Fuentes, Santos-Iglesias, \& Sierra, 2014). Cabe mencionar que se han encontrado diferencias entre los niveles de satisfacción sexual de los individuos que practican el sexo casual y los de quienes que están comprometidos en una relación. Tanto hombres como mujeres han informado niveles más altos de satisfacción sexual al tener una relación estable (Mark, Garcia, \& Fisher, 2015).

Por otra parte, algunos estudios de satisfacción sexual han reportado discrepancias al explorar las variables sociodemográficas edad, orientación sexual y nivel de educación. La edad avanzada está relacionada con una menor actividad sexual y, por consiguiente, con una disminución en la satisfacción sexual (De Ryck, Van Laeken, Nöstlinger, Platteau, \& Colebunders, 2012; Lindau \& Gavrilova, 2010). Otras variables asociadas a la edad son la presencia de enfermedades crónicas y la aparición de disfunciones sexuales (Sierra, Vallejo-Medina, Santos-Iglesias, \& Lameiras-Fernández, 2012; Trompeter, Bettencourt, \& Barrett-Connor, 2012). En términos de la orientación sexual, algunos estudios asocian la homosexualidad con un aumento en la satisfacción sexual (Henderson, Lehavot, \& Simoni, 2009), mientras que los hallazgos de otras investigaciones han revelado una mayor satisfacción sexual en hombres heterosexuales (Dixon, 1985). Niveles más altos de satisfacción sexual también han sido correlacionados con un alto nivel de educación (Carpenter, Nathanson, \& Kim, 2009) y 
menor cantidad de parejas sexuales (Heiman et al., 2011).

Asimismo, se han realizado investigaciones para asociar la importancia de la imagen corporal con el funcionamiento sexual. Estos estudios han sido mayormente enfocados en mujeres dado el énfasis otorgado a la imagen de la mujer, particularmente en las culturas occidentales. Los hallazgos de varios estudios han confirmado que las mujeres que se sienten más cómodas con su imagen corporal revelan niveles más altos de experiencia sexual, mayor frecuencia en la actividad sexual y mayor disposición para iniciar los encuentros sexuales, comparado a las que se sienten menos satisfechas con su imagen (Ackard, Kearney-Cooke, \& Peterson, 2000; Schooler, Ward, Merriwether, \& Caruthers, 2005). Una percepción negativa de la imagen corporal en la mujer también ha sido relacionada con la evitación del encuentro sexual. El estudio de La Rocque y Cioe (2011) evaluó esta asociación y confirmó que las mujeres con una autoimagen negativa presentaron una mayor evasión ante la actividad sexual. Del mismo modo, el que las mujeres no se sientan a gusto con su imagen contribuye a la disminución del placer, los orgasmos y la satisfacción sexual (Sanchez \& Kiefer, 2007; Yamamiya, Cash, \& Thompson, 2006).

Cabe mencionar que el orgasmo y su consistencia han sido utilizados como medidas en los estudios de satisfacción, ya que ambas han ofrecido datos cuantificables y válidos a través de los participantes (Darling et al., 1991; Haavio-Mannila \& Kontula, 1997; Young, Denny, Young, \& Luquis, 2000). Mientras que el orgasmo puede proveer un cierto tipo de resultado acerca de la experiencia sexual, es solo una faceta de esta y no necesariamente está asociado a la satisfacción en todos los individuos (Haavio-Mannila \& Kontula, 1997). De acuerdo con Hurlbert et al. (1993), el deseo, la excitación y la consistencia del orgasmo están vinculados a una mayor satisfacción sexual. No obstante, las mujeres pueden expresar niveles más bajos de frecuencia orgásmica e incluso sentirse satisfechas sexualmente (McClelland, 2009). Algunos individuos también han revelado mayores niveles de orgasmo durante el coito cuando tienen un encuentro sexual con sus parejas, comparado con los que tienen encuentros sexuales fuera de relaciones comprometidas (Armstrong, England, \& Fogarty, 2012; Mark et al., 2015). La frecuencia del coito y la libido también han correlacionado positivamente con el constructo satisfacción sexual (Daker-White \& Donovan, 2002; Young et al., 1998).

Escala de Satisfacción Sexual Subjetiva (ESSS) Versión original

González-Rivera et al. (2017) desarrollaron y validaron la versión original de la ESSS. Esta escala fue desarrollada por sus investigadores con el propósito de medir el constructo satisfacción sexual desde un modelo de cuatro factores: $v a$ loración subjetiva, aspecto emocional, ejecución sexual y autoimagen. La escala fue administrada en una muestra de 615 adultos puertorriqueños de entre 20 y 75 años. Las puntuaciones de la ESSS tienen un rango de 20 a 80 , siendo las mayores puntuaciones las que revelan una mayor satisfacción sexual en la persona. La versión original de la ESSS consta de 20 reactivos (5 en cada dimensión), los cuales reflejan indicadores adecuados de validez y confiabilidad. Para examinar la validez del instrumento se realizaron análisis correlacionales entre la ESSS y los cuatro factores y se obtuvieron resultados aceptables. Los análisis de consistencia interna para determinar la confiabilidad de la escala original mostraron índices adecuados de .91 y .89, según las pruebas alfa de Cronbach y Spearman-Brown. 
El objetivo principal de este proyecto es desarrollar una versión breve de la ESSS que facilite la tarea investigativa de temas asociados al conflicto y la satisfacción en las relaciones de pareja. Para ello, se llevaron a cabo dos estudios independientes. En el Estudio 1, con la intención de desarrollar una versión breve de la ESSS, se analiza la estructura factorial de la escala mediante un análisis factorial exploratorio (AFE) con criterios estrictos de selección, se evalúa la confiabilidad del instrumento y se examina la capacidad de discriminación de los ítems. En el Estudio 2, se analiza la validez de constructo de la versión breve de la escala mediante un análisis factorial confirmatorio (AFC) con ecuaciones estructurales, se evalúa la confiabilidad compuesta y se examina la validez concurrente y convergente del instrumento. En los dos estudios se utiliza un diseño de investigación instrumental (Montero \& León, 2007). Ambos estudios fueron aprobados por el Comité para la Ética en la Investigación (IRB) de la Universidad Carlos Albizu en San Juan, Puerto Rico.

\section{Estudio 1}

Método

Participantes

Para participar del Estudio 1, establecimos los siguientes criterios de inclusión para adecuar la muestra al propósito del estudio (validar un instrumento que facilite estudiar temas asociados al conflicto y la satisfacción en las relaciones de pareja): tener 21 años o más y estar en una relación de pareja (matrimonio, convivencia libre o noviazgo). Se trabajó con una muestra no probabilística constituida por 200 participantes, de los cuales 157 eran mujeres (78.5\%) y 43 hombres (21.5\%). El rango de edad estaba entre los $21 \mathrm{y}$ los 60 años, de los cuales el 31.5\% $(\mathrm{n}=63)$ tenía entre 21 y 30 años, el $30.5 \%(\mathrm{n}=61)$ entre 31 y 40 años, el 34\% $(n=68)$ entre 41 y 50 años y el $4 \%(n=8)$ tenía más de 51 años. En cuanto al estado civil, el $69.5 \%(\mathrm{n}=174)$ estaba casado legalmente, el $24 \%(n=48)$ convivía con su pareja sin nupcias y el $6.5 \%(n=13)$ era soltero, pero en una relación de noviazgo. En términos de ingresos económicos, el 44.5\% $(\mathrm{n}=89)$ generaba ingresos menores a $\$ 25^{\prime} 000$, mientras que el $32.5 \%(\mathrm{n}=$ $65)$ entre $\$ 26^{\prime} 000$ y $\$ 50 ’ 000$, y el $23 \%(n=46)$ ingresos superiores a $\$ 50 ’ 000$. Por último, el 98\% $(\mathrm{n}=196)$ se identificó como heterosexual, 1\% (n $=2)$ como bisexual y $1 \%(n=2)$ como lesbiana.

\section{Instrumento}

La Escala de Satisfacción Sexual Subjetiva (ESSS), desarrollada por González-Rivera et al. (2017), se compone de 20 ítems organizados en una escala tipo Likert de cuatro puntos que va de (1) Totalmente en desacuerdo a (4) Totalmente de acuerdo. La escala consta de cuatro factores: valoración subjetiva, aspecto emocional, ejecución sexual y autoimagen. El rango posible es de 20 a 80 puntos. A mayor puntuación, mayor satisfacción sexual exhibe la persona $(\alpha=.90)$.

\section{Procedimientos generales}

La recopilación de datos se llevó a cabo mediante el uso de cuestionarios a través de la plataforma PsychData utilizando como método de reclutamiento las redes sociales Facebook, Twitter, Google + y WhatsApp, entre otras. Esto permitió un efecto bola de nieve en las redes sociales. Una vez que accedían a la encuesta en línea, los participantes leían el consentimiento informado, en el cual se notificaba el propósito del estudio, los criterios de inclusión, su naturaleza voluntaria 
y los posibles riesgos y beneficios, así como su derecho a retirarse del estudio en cualquier momento. Además, se les notificaba sobre la duración de su participación y su derecho a obtener los resultados del estudio una vez culminara la investigación. Para garantizar la privacidad y confidencialidad de los participantes, los cuestionarios se completaban de forma anónima y los participantes tenían la posibilidad de imprimir una copia del consentimiento informado. Los datos fueron analizados en el programa estadístico IBM SPSS v. 24 (IBM Corp., 2016).

\section{Resultados}

Análisis factorial

En el primer estudio se realizó un AFE utilizando el método de extracción de máxima verosimilitud con rotación oblicua. Como criterios de aceptación, consideramos aquellos ítems con una carga factorial mayor a .50 en un solo factor (Stevens, 2002). Todos los ítems que no cumplieran este criterio serían eliminados de la versión corta del instrumento. Los resultados mostraron una solución factorial que explica el 64\% de la varianza de los datos $\left(\mathrm{KMO}=.89 ; \chi^{2}=1787.963_{(190)}\right.$, $p<.001)$. En este primer análisis, de los 20 ítems de la escala original, se eliminaron siete ítems por no cumplir con los criterios de selección (ítems 6 , $10,11,12,16,19$ y 20). La versión de 13 ítems fue sometida a un segundo AFE que mostró una solución factorial que explica el $65 \%$ de la varianza de los datos $\left(\mathrm{KMO}=.90 ; \chi^{2}=1253.05_{(78)}, p<\right.$ $.001)$. En esta ocasión, dos ítems no cumplieron con los criterios de selección, por lo cual fueron eliminados (ítems 4 y 15).

Un tercer AFE con la versión de 11 ítems mostró una solución factorial que explica el $62 \%$ de la varianza de los datos $\left(\mathrm{KMO}=.92 ; \chi^{2}\right.$ $\left.=1082.998_{(55)}, p<.001\right)$. En esta ocasión, otros cuatro ítems no cumplieron con los criterios de selección (ítems 7, 8, 13 y 18). La última versión de siete ítems fue sometida a un AFE. Los resultados confirmaron que estos siete ítems cargaron en un solo factor, cumplieron con los criterios de selección y explicaron el $65 \%$ de la varianza de los datos $\left(\mathrm{KMO}=.92 ; \chi^{2}=792.128_{(21)}, p<.001\right)$. En la Tabla 1 se presentan las cargas factoriales obtenidas por los siete ítems de la versión final.

\section{Análisis de Discriminación}

Una vez que se obtuvo una versión reducida de la escala, se llevó a cabo un análisis de reactivos para calcular los índices de discriminación de los siete ítems de la versión breve mediante el método de correlación del ítem con el total de la prueba $\left(r_{\text {bis }}\right)$. Los resultados mostraron índices de discriminación que fluctuaban de .67 a .81, de manera que todos los ítems obtuvieron índices de discriminación mayores a .30, como recomienda Kline (2005). La Tabla 1 presenta los índices de discriminación de los siete ítems de la escala.

\section{Análisis de Confiabilidad}

La versión breve de la escala fue sometida a un análisis de consistencia interna para determinar el índice de confiabilidad de la escala. Para ello utilizamos dos métodos: el coeficiente alfa de Cronbach y división en mitades de SpearmanBrown. Los resultados mostraron un coeficiente alfa de .91 y de .87 para la prueba SpearmanBrown. La Tabla 2 presenta el alfa de Cronbach, alfa de Cronbach estandarizado, el coeficiente de la prueba Spearman-Brown, la media y la desviación estándar de la Versión Breve de la Escala de Satisfacción Sexual Subjetiva (ESSS-B). 
Tabla 1

Análisis de factores exploratorios e índices de discriminación de los ítems.

\begin{tabular}{|c|c|c|}
\hline Ítems & $r_{b i s}$ & 1 \\
\hline 1. Considero que mi vida sexual es muy excitante. & .77 & .83 \\
\hline 2. Luego de tener relaciones sexuales me siento pleno. & .69 & .73 \\
\hline 3. Estoy satisfecho con la cantidad de sexo que practico a la semana. & .73 & .79 \\
\hline 5. Mi vida sexual es divertida. & .81 & .86 \\
\hline 9. A mi vida sexual le falta calidad. & .67 & .70 \\
\hline 14. Me siento satisfecho con la frecuencia de mis orgasmos. & .67 & .70 \\
\hline 17. Me siento cómodo con la calidad de sexo que practico. & .71 & .74 \\
\hline
\end{tabular}

Nota. $r_{\text {bis }}=$ índice de discriminación del ítem; 1 = cargas factoriales. $(\mathrm{n}=200)$.

Tabla 2

Media, desviación estándar y coeficientes de confiabilidad.

\begin{tabular}{llllll}
\hline Factor & M & DE & $\alpha$ & $\alpha_{\text {est }}$ & $\begin{array}{c}\text { Spearman- } \\
\text { Brown }\end{array}$ \\
\hline ESSS- B (siete ítems) & 20.56 & 5.33 & .91 & .91 & .87 \\
\hline
\end{tabular}

Nota. $\mathrm{M}=$ media; $\mathrm{DE}=$ desviación estándar; $\alpha=$ alfa de Cronbach; $\alpha_{\mathrm{est}}=$ alfa de Cronbach estandarizado. $(\mathrm{n}=200)$.

\section{Estudio 2}

Método

Participantes

Para participar del Estudio 2, establecimos los mismos criterios de inclusión que para el estudio 1: tener 21 años o más y estar en una relación de pareja, dado que es requerimiento de uno de los instrumentos administrados (Escala de Evaluación de la Relación de Pareja). Se trabajó con una muestra no probabilística constituida por 275 participantes. De estos, 212 eran mujeres (77.1\%) y 63 hombres (22.9\%). El rango de edad estaba entre los 21 y los 62 años, con una edad promedio de $32.80(\mathrm{DE}=6.99)$. En cuanto al estado civil, el 48.4\% $(n=133)$ estaba casado legalmente y el $51.6 \%(n=142)$ convivía con su pareja sin nupcias. En términos de la preparación académica, el 38.9\% $(\mathrm{n}=107)$ poseía un bachillerato, el 21.5\% $(\mathrm{n}=59)$ poseía un grado de maestría, el $25.8 \%(\mathrm{n}=71)$ tenía un grado técnico o grado asociado, el 8.4\% $(\mathrm{n}=23)$ completó únicamente la escuela superior y el $5.5 \%(\mathrm{n}=15)$ contaba con un grado doctoral. La mayoría de la muestra $(64.7 \%, \mathrm{n}=178)$ generaba ingresos anuales menores a $\$ 25^{\prime} 000$, el 27.3\% $(\mathrm{n}=75)$ entre $\$ 26^{\prime} 000$ y $\$ 50 ’ 000$ y el $8 \%(n=22)$ ingresos superiores a $\$ 50$ ’000 anuales. Por último, el 100\% $(\mathrm{n}=275)$ se identificó como heterosexual.

\section{Instrumentos}

En el Estudio 2 se utilizaron tres instrumentos: la Escala de Satisfacción Sexual Subjetiva (ESSS; véase descripción en el Estudio 1), la versión breve de la Escala de Satisfacción Sexual Subjetiva (ESSS-B) y la Escala de Evaluación de 
la Relación de Pareja. La ESSS-B, validada en el Estudio 1, se compone de 7 ítems organizados en una escala tipo Likert de cuatro puntos que va de (1) Totalmente en desacuerdo a (4) Totalmente de acuerdo. El rango posible es de 7 a 28 puntos. A mayor puntuación, mayor satisfacción sexual reporta la persona $(\alpha=.91)$.

Por su parte, la Escala de Evaluación de la Relación de Pareja (Hendrick, 1988) mide de forma global los sentimientos, pensamientos o conductas en la relación de pareja (por ejemplo, ¿Qué tanto tu pareja satisface tus necesidades?). Consta de siete ítems con una escala de respuesta de cinco puntos que fluctúan de Nada a Totalmente. El rango posible es de 7 a 35 puntos. A mayor puntaje, mayor satisfacción y calidad en la relación de pareja exhibe la persona $(\alpha=.92)$.

\section{Procedimientos generales}

La recopilación de datos se llevó a cabo mediante el uso de cuestionarios a través de la plataforma PsychData utilizando como método de reclutamiento las redes sociales Facebook, Twitter, Google + y WhatsApp, entre otras. Esto permitió un efecto bola de nieve en las redes sociales. Una vez que accedían a la encuesta en línea, los participantes leían el consentimiento informado, en el cual se notificaba el propósito del estudio, los criterios de inclusión, su naturaleza voluntaria y los posibles riesgos y beneficios, así como su derecho a retirarse del estudio en cualquier momento. Además, se les notificaba sobre la duración de su participación y su derecho a obtener los resultados del estudio una vez que culminara la investigación. Para garantizar la privacidad y confidencialidad de los participantes, los cuestionarios se completaron de forma anónima y los participantes tenían la posibilidad de imprimir una copia del consentimiento informado. Los datos fueron analizados en el programa estadístico STATA 15 (StataCorp., 2017).

\section{Resultados \\ Análisis descriptivos de los ítems}

En primer lugar, se calcularon las medias y desviaciones estándar para cada ítem de la versión breve para analizar la distribución de la escala. Las medias de los ítems fluctuaron entre 2.44 y 2.99, con desviaciones estándar que fluctuaron entre .90 y 1.13 . Como muestra la Tabla 3, las pruebas de normalidad Kolmogorov-Smirnov y Shapiro-Wilk evidencian que los datos no se distribuyen de forma normal $(p<.001)$. Por la falta de normalidad en los datos, utilizamos las correcciones de Satorra y Bentler (2001) para calcular el ajuste de los modelos de ecuaciones estructurales, ya que la no normalidad de los datos altera los errores de estimación y el ajuste global del modelo.

\section{Validez de Constructo}

Se realizó un AFC con ecuaciones estructurales utilizando el método de estimación de máxima verosimilitud. Para evaluar el ajuste del modelo se utilizaron los siguientes índices de bondad de ajuste y los criterios de Byrne (2010): Chi-cuadrado $\left(\chi^{2}\right)$, error medio cuadrático de aproximación (RMSEA; valores iguales o menores a .08 indican un buen ajuste), índice TuckerLewis (TLI) y el índice de ajuste comparativo (CFI). Para que exista un buen ajuste del modelo, los valores sugeridos de CFI y TLI deben ser mayores o iguales que 95 (Byrne, 2010). Los resultados arrojaron un buen ajuste para el modelo conformado por un solo factor latente que recogía los siete ítems de la escala (véase Figura 1), 
Tabla 3

Estadísticos de descriptivos y de distribución para los ítems de la escala.

\begin{tabular}{cccccccc}
\hline Ítem & Media & $\begin{array}{c}\text { Desviación } \\
\text { Estándar }\end{array}$ & Asimetría & Curtosis & $\begin{array}{c}\text { Kolmogo- } \\
\text { rov-Smirnov }\end{array}$ & Shapiro-Wilk & $\boldsymbol{r}_{\text {bis }}$ \\
\hline 1 & 2.68 & 0.94 & -0.30 & -0.77 & .25 & .870 & .78 \\
2 & 2.99 & 0.90 & -0.55 & -0.52 & .23 & .845 & .75 \\
3 & 2.44 & 1.00 & 0.09 & -1.06 & .21 & .877 & .73 \\
5 & 2.61 & 0.97 & -0.06 & -0.97 & .20 & .878 & .80 \\
9 & 2.55 & 1.13 & 0.02 & -1.39 & .21 & .847 & .71 \\
14 & 2.66 & 1.01 & -0.26 & -1.02 & .23 & .869 & .69 \\
17 & 2.75 & 0.96 & -0.26 & -0.90 & .22 & .871 & .73 \\
\hline
\end{tabular}

Nota. $r_{\text {bis }}=$ índice de discriminación del ítem; error estándar de la asimetría $=.15$; error estándar de la curtosis $=.29$; grados de libertad Kolmogorov-Smirnov y Shapiro-Wilk $=275$; todos los valores $p<.001$.

$\chi^{2}=44.845_{(14)}, p<.001 ;$ RMSEA $=.09 ; \mathrm{CFI}=$ $.97, \mathrm{TLI}=.96 ; \chi^{2}$ corregido $=36.693_{(14)}, p<.001$; RMSEA corregido $=.08 ;$ CFI corregido $=.98$, TLI corregido $=.97$. A su vez, se examinaron los coeficientes de regresión de cada ítem; se esperaba que cada uno de estos fuese mayor a .65, según recomiendan Schumaker y Lomax (2010). Los coeficientes de regresión fluctuaron entre .71 y .86 (véase Tabla 4). Luego, se analizó la confiabilidad compuesta del modelo unidimensional de la escala. Los análisis revelaron un índice de confiabilidad compuesta de .92 y una varianza media explicada de .61, superando el valor mínimo recomendado por la literatura (Raykov \& Shrout, 2002).

\section{Validez de Criterio}

Con el fin de evaluar la validez concurrente y convergente de la ESSS-B, se analizaron las correlaciones con la versión larga del instrumento y una medida de satisfacción en la relación de pareja. Como se puede observar en la Tabla 5, todas las correlaciones fueron significativas. Según lo esperado, las correlaciones entre las dos versio-

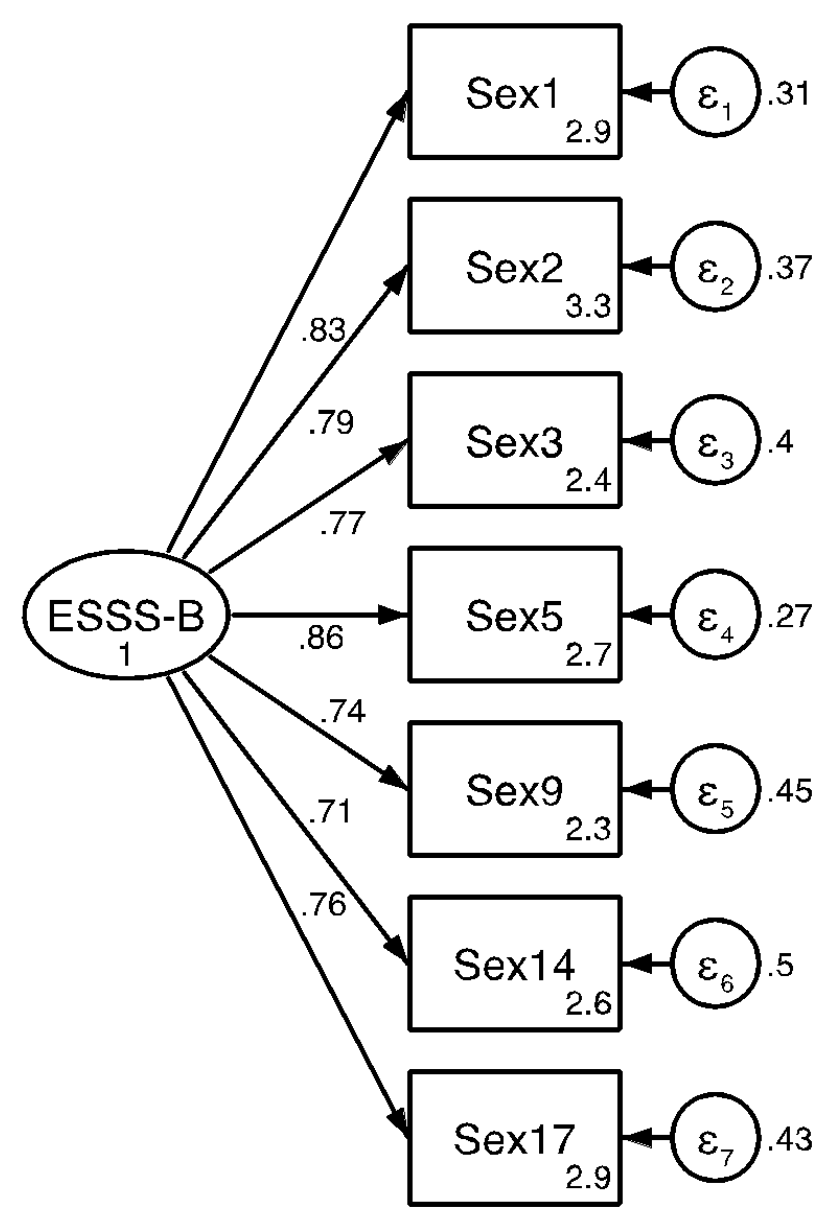

Figura 1

Modelo unidimensional de la Versión Breve de la Escala de Satisfacción Sexual Subjetiva (ESSS-B). 
Tabla 4

Estimados estandarizados de regresión y sus intervalos de confianza.

\begin{tabular}{llll}
\hline \multicolumn{1}{c}{ Ítems } & $\boldsymbol{\beta}$ & $\boldsymbol{p}$ & I.C. 95\% \\
\hline 1. Considero que mi vida sexual es muy excitante. & .83 & $<.001$ & {$[.79, .88]$} \\
2. Luego de tener relaciones sexuales me siento pleno. & .79 & $<.001$ & {$[.63, .76]$} \\
3. Estoy satisfecho con la cantidad de sexo que practico a la semana. & .77 & $<.001$ & {$[.75, .84]$} \\
5. Mi vida sexual es divertida. & .86 & $<.001$ & {$[.80, .92]$} \\
9. A mi vida sexual le falta calidad. & .74 & $<.001$ & {$[.66, .82]$} \\
14. Me siento satisfecho con la frecuencia de mis orgasmos. & .71 & $<.001$ & {$[.64, .78]$} \\
17. Me siento cómodo con la calidad de sexo que practico. & .76 & $<.001$ & {$[.70, .82]$} \\
\hline
\end{tabular}

Nota. $\beta=$ estimados estandarizados de regresión; $p=$ significación; I.C. $95 \%$ = intervalos de confianza de los coeficientes de regresión.

Tabla 5

Correlaciones entre las escalas, sus medias, desviaciones estándar y alfa de Cronbach.

\begin{tabular}{|c|c|c|c|c|c|}
\hline & $\alpha$ & $\mathbf{M}$ & DE & 1 & 2 \\
\hline 1. ESSS-B & .91 & 18.68 & 5.64 & & \\
\hline 2. ESSS (versión larga) & .90 & 58.72 & 10.81 & .89 & \\
\hline 3. Satisfacción en la Relación & .90 & 25.59 & 5.73 & .68 & .61 \\
\hline
\end{tabular}

Nota. Todas las correlaciones significativas a .001. $(\mathrm{n}=342) ; \alpha=$ alfa de Cronbach.

nes del instrumento fueron significativamente altas y positivas. Asimismo, la correlación entre la ESSS-B y la satisfacción en la relación de pareja fue positiva.

\section{Discusión}

El presente proyecto de investigación tuvo como propósito desarrollar una versión breve de la ESSS que facilite la tarea investigativa de temas asociados al conflicto y a la satisfacción en las relaciones de pareja. Para ello, se analizaron las propiedades psicométricas de la ESSS utilizando criterios estrictos de selección de ítems en dos muestras de adultos puertorriqueños. A partir de los resultados obtenidos en ambos estudios, se obtuvo una versión breve del instrumento con siete ítems (ESSS-B) que posee adecuadas características psicométricas para examinar de manera subjetiva y general la satisfacción sexual de una persona. Asimismo, los índices de confiabilidad obtenidos sugieren, según lo establece DeVellis (2017), que la ESSS-B cuenta con la suficiente consistencia interna para ser utilizada como instrumento de medición científica en investigaciones en Puerto Rico e incluso como instrumento de avalúo general en los procesos de psicoterapia y consejería.

De forma general, el adicionamiento del AFE del Estudio 1 con el AFC del Estudio 2 demuestra que la versión unidimensional de la ESSS-B presenta un ajuste satisfactorio con los datos. Esto es congruente con las tendencias actuales del estudio de la satisfacción sexual que favorecen la medición de este constructo desde una 
manera subjetiva basada en el juicio global de las experiencias vividas por las personas (Lawrance \& Byers, 1995; McClelland, 2010). En otras palabras, las puntuaciones obtenidas en la ESSS-B cubren varios aspectos de la experiencia sexual de una forma global, sobre los cuales la persona emite un juicio valorativo, ya sea positivo (satisfacción) o negativo (insatisfacción). Asimismo, pueden considerarse como una actitud de favorabilidad o desfavorabilidad hacia su actividad sexual al contrastarlas con sus expectativas individuales.

En cuanto a la confiabilidad del instrumento, se obtuvieron índices superiores a los recomendados por la literatura (Campo-Arias \& Oviedo, 2008; Kline, 2005), lo cual sugiere que la versión final de la escala es un instrumento estable, reproducible y consistente en la medida de la satisfacción sexual. De igual forma, las correlaciones de cada ítem con la puntuación total manifiestan una consistencia interna notable. Esto sugiere que los ítems de la versión final discriminan adecuadamente y son capaces de diferenciar personas con distintos niveles de satisfacción sexual.

Por su parte, la correlación moderadamente alta de la ESSS-B con la medida de satisfacción en la pareja aporta evidencia sobre la validez convergente de la escala y concuerda con los patrones de correlaciones obtenidos en otras investigaciones (Monteiro-Pascoal et al., 2014; Sprecher \& Cate, 2004; Stephenson et al., 2011). De igual forma, la correlación alta de las dos versiones del instrumento confirma que la versión ESSS-B tiene la capacidad de cuantificar de forma significativa y adecuada el mismo constructo que evalúa la ESSS. Esto demuestra que la ESSS-B es una medida confiable de satisfacción sexual y aporta evidencia a su validez concurrente.

En cuanto a las implicaciones prácticas del estudio, se demostró que la ESSS-B es útil para el desarrollo de nuevas investigaciones en el campo de la psicología y la sexualidad en Puerto Rico. En términos de conveniencia, la principal fortaleza de la escala es su lenguaje sencillo y su brevedad, lo que la consolida como un instrumento de fácil administración e interpretación. En este sentido, la ESSS-B se constituye como una herramienta práctica y efectiva en el quehacer investigativo y académico de la psicología. Las puntuaciones de la ESSS-B se calculan mediante la sumatoria de los siete ítems para obtener un índice general. Puntuaciones entre 25 y 28 se consideran indicadores de una alta satisfacción sexual; puntuaciones entre 15 y 24 se consideran indicadores de una satisfacción sexual promedio; y puntuaciones entre 7 y 14 indican una baja satisfacción sexual.

Al igual que toda investigación, nuestro estudio no está exento de limitaciones. Primero, la muestra fue reunida de forma accidental y no fue aleatoria (muestra no probabilística). Segundo, no se pudo establecer la confiabilidad del instrumento a través del tiempo, solo se pudo hacer a través de sus elementos. No obstante, las técnicas avanzadas utilizadas en el estudio brindan robustez empírica a nuestros resultados. Tercero, las muestras utilizadas en ambos estudios están integradas por personas con parejas (casados, unión libre, noviazgo), por lo cual no sabemos cómo se comporta la escala en personas sin pareja que mantienen una vida sexual activa. Cuarto, el 98\% de los participantes del Estudio 1 y la totalidad de la muestra del Estudio 2 se identificaron como heterosexuales, por lo cual nuestros hallazgos y conclusiones se limitan a personas heterosexuales con pareja. Por último, el procedimiento para recoger los datos no fue estandarizado, lo que puede afectar las medias del estudio y aumentar el error estándar de medición.

Para futuros estudios, se recomienda examinar la confiabilidad temporal a través de la técnica de test-retest y realizar el proceso de validación 
cruzada. A su vez, se recomienda que se valide la ESSS-B en otras poblaciones latinoamericanas para examinar sus propiedades psicométricas en distintos contextos nacionales e internacionales. Por último, se sugiere replicar estos resultados en muestras más amplias, equilibradas por sexo y que incluyan un número considerable de participantes de la comunidad LGBT.

En síntesis, las propiedades psicométricas obtenidas demuestran que la ESSS-B refleja indicadores adecuados de validez y confiabilidad. El hecho de utilizar dos muestras independientes para validar y examinar la ESSS-B da un valor añadido al estudio. Se concluye que la ESSS-B es un excelente instrumento de medición para futuras investigaciones en Puerto Rico y recomendamos su validación en otros países latinoamericanos.

\section{Referencias}

Ackard, D. M., Kearney-Cooke, A., \& Peterson, C. B. (2000). Effects of body image and self-image on women's sexual behaviors. International Journal of Eating Disorders, 28(4), 422-429. doi: 10.1002/1098-108x(200012)28:4<422::aid-eat10>3.0.co;2-1

Ahumada, S., Lüttges, C., Molina, T., \& Torres, S. (2014). Satisfacción sexual: Revisión de los factores individuales y de pareja relacionados. Revista Hospital Clínico Universidad de Chile, 25(4), 278-284. Recuperado de https://www.redclinica.cl

Armstrong, E. A., England, P., \& Fogarty, A. C. K. (2012). Accounting for women's orgasm and sexual enjoyment in college hookups and relationships. American Sociological Review, 77(3), 435-462. doi: 10.1177/0003122412445802

Byrne, B. M. (2010). Structural equation modeling with AMOS: Basic concepts, applications, and programming ( $2^{a}$ ed.). New York, NY: Taylor \& Francis Group. doi: https://www.taylorfrancis.com/ books/9781410600219

Campo-Arias, A., \& Oviedo, H. C. (2008). Propiedades psicométricas de una escala: La consistencia interna. Revista de Salud Pública, 10(5), 831-839. doi: 10.1590/s0124-00642008000500015

Carpenter, L. M., Nathanson, C. A., \& Kim, Y. J. (2009). Physical women, emotional men: Gender and sexual satisfaction in midlife. Archives of Sexual Behavior, 38(1), 87-107. doi: 10.1007/s10508-007-9215-y

Daker-White, G., \& Donovan, J. (2002). Sexual satisfaction, quality of life and the transaction of intimacy in hospital patients' accounts of their (hetero)sexual relationships. Sociology of Health and Illness, 24(1), 89-113. doi: 10.1111/1467-9566.00005

Darling, C. A., Davidson, J. K., \& Jennings, D. A. (1991). The female sexual response revisited: Understanding the multiorgasmic experience in women. Archives of Sexual Behavior, 20(6), 527-540. doi: 10.1007/ BF01550952

Davison, S. L., Bell, R. J., LaChina, M., Holden, S. L., \& Davis, S. R. (2009). The relationship between self-reported sexual satisfaction and general well-being in women. The Journal of Sexual Medicine, 6(10), 2690-2697. doi: 10.1111/j.1743-6109.2009.01406.x

De Ryck, I., van Laeken, D., Nöstlinger, C., Platteau, T., \& Colebunders, R. (2012). Sexual satisfaction among men living with HIV in Europe. AIDS and Behavior, 16(1), 225-230. doi: 10.1007/s10461-011-9987-X

DeLamater, J. (1991). Emotions and sexuality. En K. McKinney \& S. Sprecher (Eds.), Sexuality in Close Relationships (pp. 49-70). Hillsdale, NJ: Lawrence Erlbaum.

DeLamater, J., \& Hyde, J. (2004). Conceptual and theoretical issues in studying sexuality in close relationships. En J. H. Harvey, A. Wenzel, \& S. Sprecher (Eds.), The Handbook of Sexuality in Close Relationships (pp. 7-30). Mahwah, NJ: Lawrence Erlbaum. doi: $10.4324 / 9781410610249$

DeRogatis, L. R. (2008). Assessment of sexual function/dysfunction via patient reported outcomes. International Journal of Impotence Research, 20(1), 
35-44. doi: 10.1038/sj.ijir.3901591

DeVellis, R. F. (2017). Scale development: Theory and applications. California, CA: Sage.

Dixon, D. (1985). Perceived sexual satisfaction and marital happiness of bisexual and heterosexual swinging husbands. Journal of Homosexuality, 11(1-2), 209222. doi: $10.1300 / J 082$ v11n01_15

González-Rivera, J. A., Veray-Alicea, J., Santiago-Santos, D., Castro-Castro, S., \& Quiñones-Soto, R. (2017). Desarrollo y validación de una escala para medir satisfacción sexual subjetiva en adultos puertorriqueños. Salud y Conducta Humana, 4(1), 52-63. Recuperado de http://rsych.com

Haavio-Mannila, E., \& Kontula, O. (1997). Correlates of increased sexual satisfaction. Archives of Sexual Behavior, 26(4), 399-419. Recuperado de https:// link.springer.com/journal/volumesAndIssues/10508

Hassebrauck, M., \& Fehr, B. (2002). Dimensions of relationship quality. Personal Relationships, 9(3), 253270. doi: 10.1111/1475-6811.00017

Heiman, J. R., Long., J. S., Smith, S. N., Fisher, W. A., Sand, M. S., \& Rosen, R. C. (2011). Sexual satisfaction and relationship happiness in midlife and older couples in five countries. Archives of Sexual Behavior, 40(4), 741-753. doi: 10.1007/s10508-010-9703-3

Henderson, A. W., Lehavot, K., \& Simoni, J. M. (2009). Ecological models of sexual satisfaction among lesbian/bisexual and heterosexual women. Archives of Sexual Behavior, 38(1), 50-65. doi: 10.1007/ s10508-008-9384-3

Hendrick, S. S. (1988). A generic measure of relationship satisfaction. Journal of Marriage and Family, 50(1), 93-98. doi: 10.2307/352430

Hurlbert, D. F., Apt, C., \& Rabehl, S. M. (1993). Key variables to understanding female sexual satisfaction: An examination of women in nondistressed marriages. Journal of Sex \& Marital Therapy, 19(2), 154-165. doi: 10.1080/00926239308404899

IBM (2016). IBM SPSS Statistics for Windows, Version 24.0. [software de cómputo]. Armonk, NY: IBM.

Kline, T. J. B. (2005). Psychological testing: A practical approach to design and evaluation. Thousand Oaks, CA: Sage. doi: 10.4135/9781483385693

La Rocque, C. L., \& Cioe, J. (2011). An evaluation of the relationship between body image and sexual avoidance. The Journal of Sex Research, 48(4), 397-408. doi: 10.1080/00224499.2010.499522

Lawrance, K. A., \& Byers, E. S. (1995). Sexual satisfaction in long-term heterosexual relationships: The interpersonal exchange model of sexual satisfaction. Personal Relationships, 2(4), 267-285. doi: 10.1111/ j.1475-6811.1995.tb00092.x

Lindau, S. T., \& Gavrilova, N. (2010). Sex, health, and years of sexually active life gained due to good health: Evidence from two US population based cross sectional surveys of ageing. BMJ: British Medical Journal, 1-11. doi: 10.1136/bmj.c810

Mark, K. P., Garcia, J. R., \& Fisher, H. E. (2015). Perceived emotional and sexual satisfaction across sexual relationship contexts: Gender and sexual orientation differences and similarities. The Canadian Journal of Human Sexuality, 24(2), 120-130. doi: 10.3138/ cjhs.242-A8

McClelland, S. I. (2009). Intimate justice: Sexual satisfaction in young adults (Disertación doctoral). Recuperado de Tesis y Disertaciones de ProQuest. (Núm. UMI 3365820)

McClelland, S. I. (2010). Intimate justice: A critical analysis of sexual satisfaction. Social and Personality Psychology Compass, 4(9), 663-680. doi: 10.1111/j.1751-9004.2010.00293.x

Metz, M. E., \& Epstein, N. (2002). Assessing the role of relationship conflict in sexual dysfunction. Journal of Sex and Marital Therapy, 28(2), 139-164. doi: 10.1080/00926230252851889

Monteiro-Pascoal, P., Narciso, I. de S. B., \& MonteiroPereira, N. (2014). What is sexual satisfaction? Thematic analysis of lay people's definitions. The Journal of Sex Research, 51(1), 22-30. doi: 10.1080/00224499.2013.815149

Montero, I., \& León, O. G. (2007). A guide for naming research studies in psychology. International Journal 
of Clinical and Health Psychology, 7(3), 847-862. Recuperado de http://www.aepc.es

Orbuch, T. L., \& Harvey, J. H. (1991). Methodological and conceptual issues in the study of sexuality in close relationships. En K. McKinney \& S. Sprecher (Eds.), Sexuality in Close Relationships (pp. 9-24). Hillsdale, NJ: Erlbaum.

Peplau, L. A., Cochran, S. D., \& Mays, V. M. (1997). A national survey of the intimate relationships of African American lesbians and gay men: A look at commitment, satisfaction, sexual behavior, and HIV disease. En B. Greene (Ed.), Ethnic and cultural diversity among lesbians and gay men. Serie Psychological perspectives on lesbian and gay issues (Vol. 3, pp. 11-38). Thousand Oaks, CA: Sage.

Raykov, T., \& Shrout, P. E. (2002). Reliability of scales with general structure: Point and interval estimation using a structural equation modeling approach. Structural Equation Modeling: A Multidisciplinary Journal, 9(2), 195-212. doi: 10.1207/S15328007SEM0902_3

Renaud, C., Byers, E. S., \& Pan, S. (1997). Sexual and relationship satisfaction in mainland China. The Journal of Sex Research, 34(4), 399-410. doi: 10.1080/00224499709551907

Rowland, D., Van Diest, S., Incrocci, L., \& Slob, A. K. (2005). Psychosexual factors that differentiate men with inhibited ejaculation from men with no dysfunction or another sexual dysfunction. The Journal of Sexual Medicine, 2(3), 383-389. doi: 10.1111/j.1743-6109.2005.20352.x

Sánchez-Fuentes, M. del M., Santos-Iglesias, P., \& Sierra, J. C. (2014). A systematic review of sexual satisfaction. International Journal of Clinical and Health Psychology, 14(1), 67-75. doi: 10.1016/ S1697-2600(14)70038-9

Sanchez, D. T., \& Kiefer, A. K. (2007). Body concerns in and out of the bedroom: Implications for sexual pleasure and problems. Archives of Sexual Behavior, 36(6), 808-820. doi: 10.1007/s10508-007-9205-0

Satorra, A., \& Bentler, P. M. (2001). A scaled difference chi-square test statistic for moment structure anal- ysis. Psychometrika, 66(4), 507-514. doi: 10.1007/ BF02296192

Schooler, D., Ward, L. M., Merriwether, A., \& Caruthers, A. S. (2005). Cycles of shame: Menstrual shame, body shame, and sexual decision-making. The Journal of Sex Research, 42(4), 324-334. doi: 10.1080/00224490509552288

Schumacker, R. E., \& Lomax, R. G. (2010). A beginner's guide to structural equation modeling ( $3^{\mathrm{a}} \mathrm{ed}$.). New York, NY: Routledge. doi: 10.4324/9780203851319

Sierra, J. C., Vallejo-Medina, P., Santos-Iglesias, P., \& Lameiras-Fernández, M. (2012). Validación del Massachusetts General Hospital-Sexual Functioning Questionnaire (MGH-SFQ) en población española. Atención Primaria, 44(9), 516-524. doi: 10.1016/j. aprim.2012.02.004

Sprecher, S. (2002). Sexual satisfaction in premarital relationships: Associations with satisfaction, love, commitment, and stability. The Journal of Sex Research, 39(3), 190-196. doi: 10.1080/00224490209552141

Sprecher, S., \& Cate, R. M. (2004). Sexual satisfaction and sexual expression as predictors of relationship satisfaction and stability. En J. H. Harvey, A. Wenzel, S. Sprecher, J. H. Harvey, A. Wenzel \& S. Sprecher (Eds.), The Handbook of Sexuality in Close Relationships (pp. 235-256). Mahwah, NJ: Lawrence Erlbaum.

StataCorp. (2017). Stata: Release 15. [software de cómputo]. College Station, TX: StataCorp. LLC.

Stephenson, K. R., Ahrold, T. K., \& Meston, C. M. (2011). The association between sexual motives and sexual satisfaction: Gender differences and categorical comparisons. Archives of Sexual Behavior, 40(3), 607618. doi: 10.1007/s10508-010-9674-4

Stevens, J. (2002). Applied multivariate statistics for the social sciences ( $4^{\mathrm{a}}$ ed.). Mahwah, NJ: Lawrence Erlbaum.

Trompeter, S. E., Bettencourt, R., \& Barrett-Connor, E. (2012). Sexual activity and satisfaction in healthy community-dwelling older women. The American Journal of Medicine, 125(1), 37-43. doi: 10.1016/j. 
amjmed.2011.07.036

Vizcarrondo-Godreau, M. C. (2001). Construcción y normalización de un inventario de satisfacción sexual dirigido a mujeres (Tesis doctoral inédita). Universidad Carlos Albizu, San Juan, Puerto Rico.

World Health Organization. (2010). Measuring sexual health: Conceptual and practical considerations and related indicators. Recuperado de http://apps.who. int

Yamamiya, Y., Cash, T. F., \& Thompson, J. K. (2006). Sexual experiences among college women: The differential effects of general versus contextual body images on sexuality. Sex Roles: A Journal of Research, 55(5-6), 421-427. doi: 10.1007/s11199-006-9096-x

Young, M., Denny, G., Luquis, R., \& Young, T. (1998). Correlates of sexual satisfaction in marriage. The Canadian Journal of Human Sexuality, 7(2), 115-127.

Young, M., Denny, G., Young, T., \& Luquis, R. (2000). Sexual satisfaction among married women. American Journal of Health Studies, 16(2), 73-84. Disponible en http://www.va-ajhs.com 